\title{
Lung Congestion as a Risk Factor in End-Stage Renal Disease
}

\author{
Carmine Zoccalia, c Rocco Tripepi ${ }^{b}$ Claudia Torino ${ }^{b} \quad$ Marianna Bellantoni ${ }^{c}$ \\ Giovanni Tripepi ${ }^{b}$ Francesca Mallamacia, c \\ ${ }^{a}$ National Research Council, Rome, and ${ }^{b}$ Clinical Epidemiology and Physiopathology of Renal Disease and \\ Hypertension Unit, and ' ${ }^{\mathrm{R}}$ enal and Transplantation Unit, Ospedali Riuniti, Reggio Calabria, Italy
}

\section{Key Words}

Cardiovascular risk - Chronic kidney disease - End-stage

renal disease · Fluid volume · Hemodialysis · Lung

congestion · Peritoneal dialysis · Volume expansion

\section{Abstract}

Background: Lung congestion is emerging as a pervasive, insidious problem in end-stage renal disease (ESRD) patients on dialysis. Summary: Chest ultrasound (US), a novel, easyto-perform, cheap technique, which is currently applied for objective monitoring of pulmonary congestion in patients with heart failure in Europe, allows reliable quantification of lung water in clinical practice. Before hemodialysis (HD), about $60 \%$ of ESRD patients displayed moderate-severe lung congestion and this alteration is frequently asymptomatic. Lung congestion is reduced but not abolished by ultrafiltration dialysis, and about one third to one fourth of patients still have excessive lung water after dialysis. Lung congestion is also prevalent in patients on peritoneal dialysis (PD), and in apparently asymptomatic HD and PD patients this alteration is strongly associated with poor physical performance. Lung water in HD patients correlates in an inverse fashion with echocardiographic parameters of systolic and diastolic function, but it is only weakly related with hydration status measured by bioimpedance analysis. Moderate-severe lung congestion is a strong predictor of death and cardiovascular events and provides prognostic information independent of NYHA class, and traditional and nontraditional risk factors in ESRD patients on HD. Key Messages: Systematic application of chest US in ESRD patients shows that hidden or clinically manifest lung congestion is exceedingly frequent in this population. This alteration largely reflects left ventricular disorders superimposed on volume overload. The clinical usefulness of systematic application of chest US in ESRD remains to be tested in a formal clinical trial.

(c) 2013 S. Karger AG, Basel

\section{Introduction}

Chronic expansion of the extracellular volume is one of the most common and time-honored derangements which compose the syndromic set of end-stage renal disease (ESRD) [1-3]. Mild-to-moderate degrees of volume expansion may go undetected or are overlooked in ESRD [4], but marked fluid overload in these patients is eventually a medical emergency demanding hospitalization and

\section{KARGER}

E-Mail karger@karger.com www.karger.com/bpu
(C) 2013 S. Karger AG, Basel

0253-5068/13/0364-0184\$38.00/0 
Fig. 1. Cardiopulmonary circulation. The figure is explained in detail in the main text.

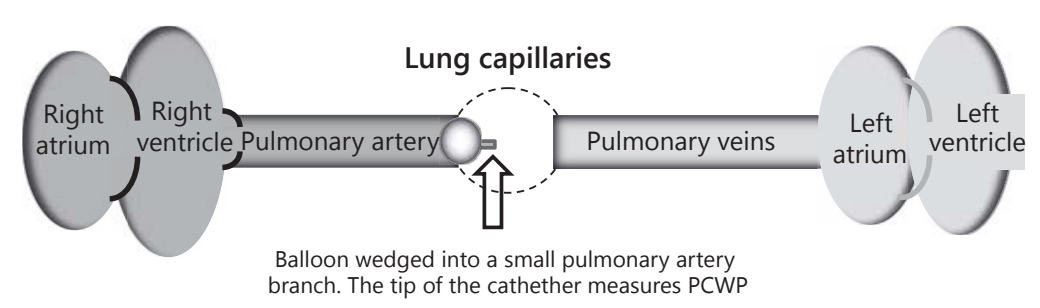

Hemodynamic congestion

(high PCWP)

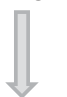

Pulmonary congestion (high lung water and high PCWP)

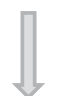

Pulmonary congestion (high lung water and normal PCWP)

Clinical congestion: dyspnea

extradialyses in these patients [5]. Even though interdialysis weight gain is not necessarily tantamount to volume expansion [6], this simple parameter has been associated with an excessive death risk [7]. Improving volume control in the dialysis population is perceived as an urgent public research issue [8].

Noninvasive methods for estimating body fluid volume and tailoring excess volume removal in ESRD have been developed over the last 20 years, including total body water, continuous hematocrit monitoring, vena cava diameter and left atrial volume measurements. Measurement of volume expansion by different parameters [9-11] predicts mortality, but the usefulness of fluid volume measurements in clinical practice still remains to be proven. The issue is of major relevance because in a randomized clinical trial that tested the effect of probing dry weight by blood volume monitoring a higher mortality and hospitalization rate was registered in the group randomized to the active arm of the trial [12].

Correction of volume expansion in the dialysis patients is difficult to achieve [4]. Due to underlying cardiomyopathy encompassing left ventricular (LV) systolic and diastolic function [13], the majority of these patients are hemodynamically fragile and may often not tolerate the standard ultrafiltration rate imposed by current dialysis schedules. However accurate and precise, estimates of fluid volume per se are not sufficient information for prescribing excess volume removal in most patients.
Extravascular lung water is a relatively small but fundamental component of body fluid volume. This component, water content in the lung interstitium, is associated with LV filling pressure estimated by pulmonary capillary wedge pressure (PCWP), i.e. the gold standard parameter for guiding fluid therapy in patients with severe heart disease [14]. Evidence that lung permeability is altered in ESRD patients was produced 50 years ago [15] implying that alterations intrinsic to the lung may contribute to the high risk of cardiopulmonary complications in this population. Recent experimental studies show that bilateral nephrectomy per se triggers lung injury via inflammatory mechanisms [16], supporting the view that lung alterations may amplify the risk of pulmonary edema in ESRD patients with volume overload and LV disorders.

Herein, we review the basic concepts of hemodynamic and pulmonary congestion and the relevance of these concepts for patients in clinical practice, and we then move to describe asymptomatic and symptomatic lung congestion in ESRD and the prognostic relevance of this disorder in the same population.

\section{Hemodynamic, Pulmonary and Clinical Congestion: Basic Concepts}

The cardiopulmonary circulation constitutes a highly integrated, strictly regulated system (fig. 1). In pulmonary capillaries, pressure is measured by right-heart 
catheterization by wedging and inflating a balloon in a small branch of the pulmonary artery and by then recording the pressure at the tip of the catheter. This approach sets the pulmonary vascular bed as a closed system. In this closed system, PCWP at the end of diastole equalizes pressure in the downstream segments of the system, i.e. the pulmonary veins, left atrium and LV. PCWP is a fundamental hemodynamic parameter which is determined by two factors: LV diastolic function and preload, which is in turn determined by circulating blood volume and venous tone. As such, PCWP is a perfect measure of the degree of filling of the most critical area of the cardiovascular system, an area extending from lung capillaries to LV. When high, PCWP indicates 'hemodynamic congestion', an alteration which can trigger redistribution of excess fluid into the lung interstitium and eventually into the alveoli, thus determining 'pulmonary congestion'. Pulmonary congestion usually underlies high PCWP and volume overload, but pulmonary congestion may be triggered by fluid redistribution rather than by high PCWP and volume overload. For example in high-altitude pulmonary edema, lung congestion is due to elevated pulmonary artery pressure caused by uneven pulmonary vasoconstriction engendered by hypoxia. Uneven vasocontriction may lead to increased perfusion of the capillaries in areas of the least arterial vasoconstriction, leading to high capillary pressure and leakage, a phenomenon likely attributable to reduced nitric oxide bioavailability, inflammation and suboptimal drainage of alveolar fluid [17]. Hemodynamic and pulmonary congestion may remain asymptomatic. When not appropriately recognized and treated, hemodynamic and pulmonary congestion eventually triggers dyspnea, i.e. 'clinical congestion', which is the main driver of hospitalization in patients with heart failure. The relevance of asymptomatic pulmonary congestion is highlighted by the observation that clinical symptoms of heart failure (i.e. clinical congestion) occur on average 4 days after evidence of hemodynamic congestion [18], and 'pulmonary congestion' detected by continuous intrathoracic impedance monitoring may be evident 2 weeks before the clinical congestion episode leading to hospitalization [19].

As will be discussed in the subsequent sections, due to volume expansion, LV disorders and lung alterations, ESRD patients are at high risk of clinical congestion. Therefore, timely detection of pulmonary congestion at a preclinical stage may be important to prevent clinical congestion, a strong driver of hospitalization in this population [5]. Thus, developing reliable methods for early detection of lung congestion is central for advancing research and knowledge on this high-risk condition in ESRD.

\section{Lung Congestion in Dialysis Patients: Risk Factors and the Problem of Timely Detection}

Pulmonary congestion and congestive heart failure are pervasive in ESRD [20]. The occurrence of symptomatic clinical pulmonary congestion by volume overload is increased in the presence of compromised LV function [21]. About half of asymptomatic ESRD patients have compromised LV systolic function [22]. In patients with LV disorders, even mild volume excess may trigger dyspnea and full-blown heart failure. The joint effect of volume overload and LV disorders on water accumulation in the lungs is epitomized by the observation that about $30 \%$ of patients entering chronic dialysis programs exhibit symptoms of heart failure, i.e. clinical congestion [23]. Experimental animals without heart disease tolerate substantial volume overload without developing relevant lung edema [24]. In contrast, pulmonary edema may occur with modest volume excess or even in the absence of overt fluid retention in patients with LV failure [18]. As alluded to before, coexistence of lung disease or lung alterations triggered by impaired renal function or exposure to dialysis membranes may further increase the risk of lung congestion in dialysis patients. Bilateral nephrectomy causes capillary leak, interstitial leukocyte infiltration and pulmonary edema in rats, a phenomenon tightly dependent on increased interleukin 6 and $1 \beta$ levels underlying the critical role of the kidney in the maintenance of serum cytokine balance and pulmonary function integrity [16]. Activation and sequestration of leukocyte neutrophils by the blood-cuprophan membrane in the dialysis filter, a rapidly reversible phenomenon which occurs at every dialysis session, may cause microvascular lung disease in hemodialysis (HD) patients [25]. Neutrophil activation and sequestration in the lungs is substantially reduced but not eliminated with synthetic and modified cellulosic membranes.

Chest ultrasound (US) is a novel method which leads to very reliable estimates of lung water in a variety of clinical conditions [26]. The basic principle of the technique is that in the presence of excessive extravascular lung water, the beam is reflected by subpleural thickened interlobular septa, a low impedance structure surrounded by air with a high acoustic mismatch. Reflection of US produces hyperechoic reverberations between the thickened 


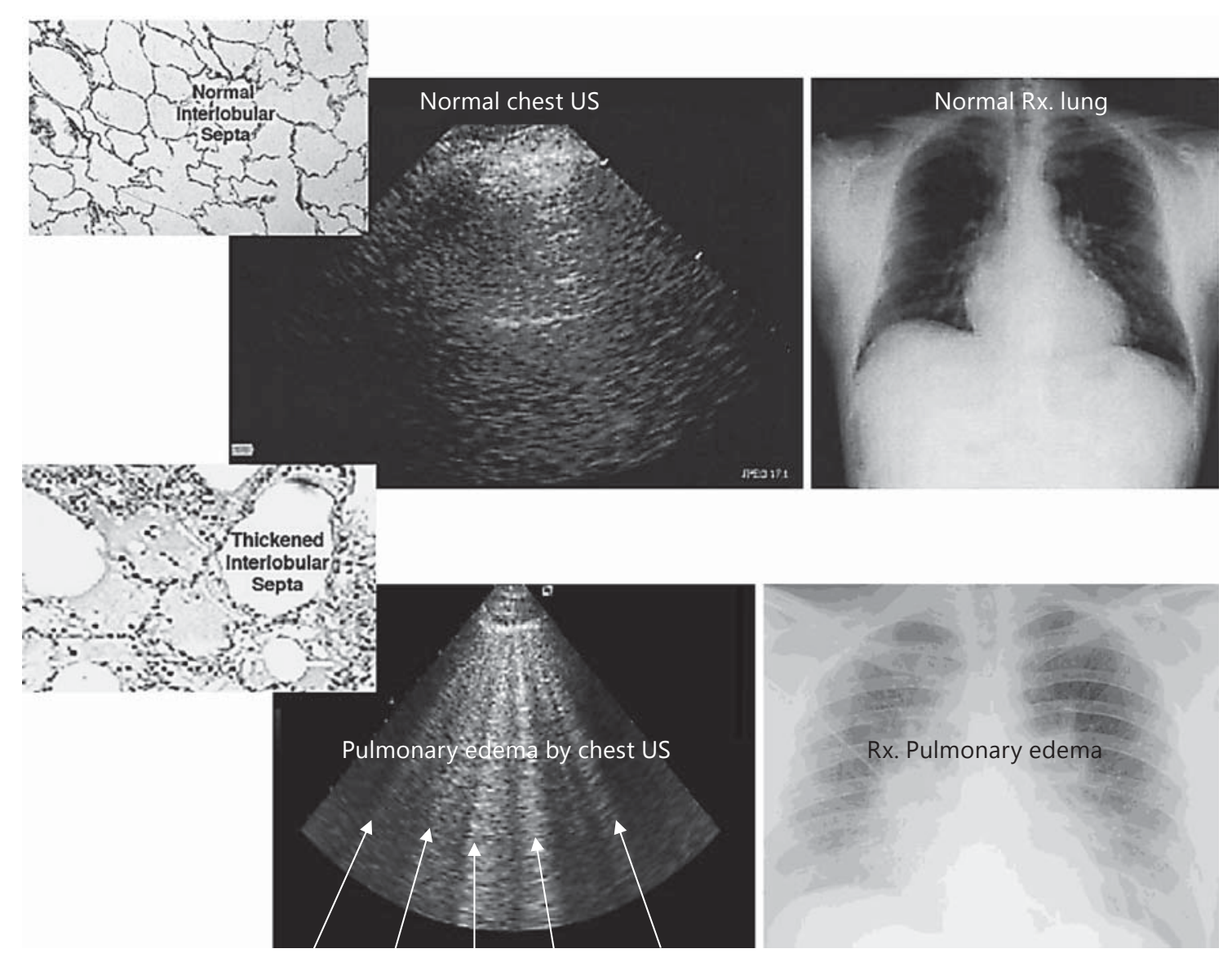

US-B lines

Fig. 2. Chest US in normal condition and in pulmonary edema. In normal condition, US beams are not or minimally reflected by the normal interalveolar septa and the scan gives a uniformly black image. In pulmonary edema, the reflection of the US by edematous, thickened alveolar septa produces characteristic reverberations that represent the US equivalent of B lines in the standard chest radiogram.

septa and the overlying pleura, which are the US equivalent of B lines seen in standard chest X-rays in patients with lung edema. These US-B lines (fig. 2 ) are easily detected with standard US probes currently applied for the study of abdominal viscera or for standard echocardiography. Chest US is very easy to learn and can be performed with virtually all US machines, including handheld US devices. Furthermore, the technique is sensitive enough for the detection of subclinical pulmonary edema in high-altitude climbers [27]. Due to its reliability and easiness of use, chest US is increasingly applied in cardiology for monitoring therapy response in patients with heart failure [28] and other conditions [26]. In a validation study in an unselected population of 75 ESRD patients on HD (26\% of them with NYHA class III-IV heart failure) [29], chest US showed good interobserver and interprobe agreement. In this study, as much as $57 \%$ of asymptomatic HD patients had moderate-severe congestion. Similar findings were registered in a proof-of-concept study aimed at testing the regression of US-B lines along with fluid removal [30]. The high frequency of lung congestion in asymptomatic patients was confirmed in a much larger, multicenter study in about 400 patients [31]. Before HD, moderate-severe lung congestion was evident in $45 \%$ of patients and very severe congestion in $14 \%$ of patients. Overall, the majority of patients (59\%) have an important degree of lung congestion before HD. Lung congestion in HD patients was very common also in a very recent study by Siriopol [32] and in another study in peritoneal dialysis (PD) patients [33]. In agreement with previous studies [29, 31], lung congestion occurred often without clinical manifestations. However, in both HD 


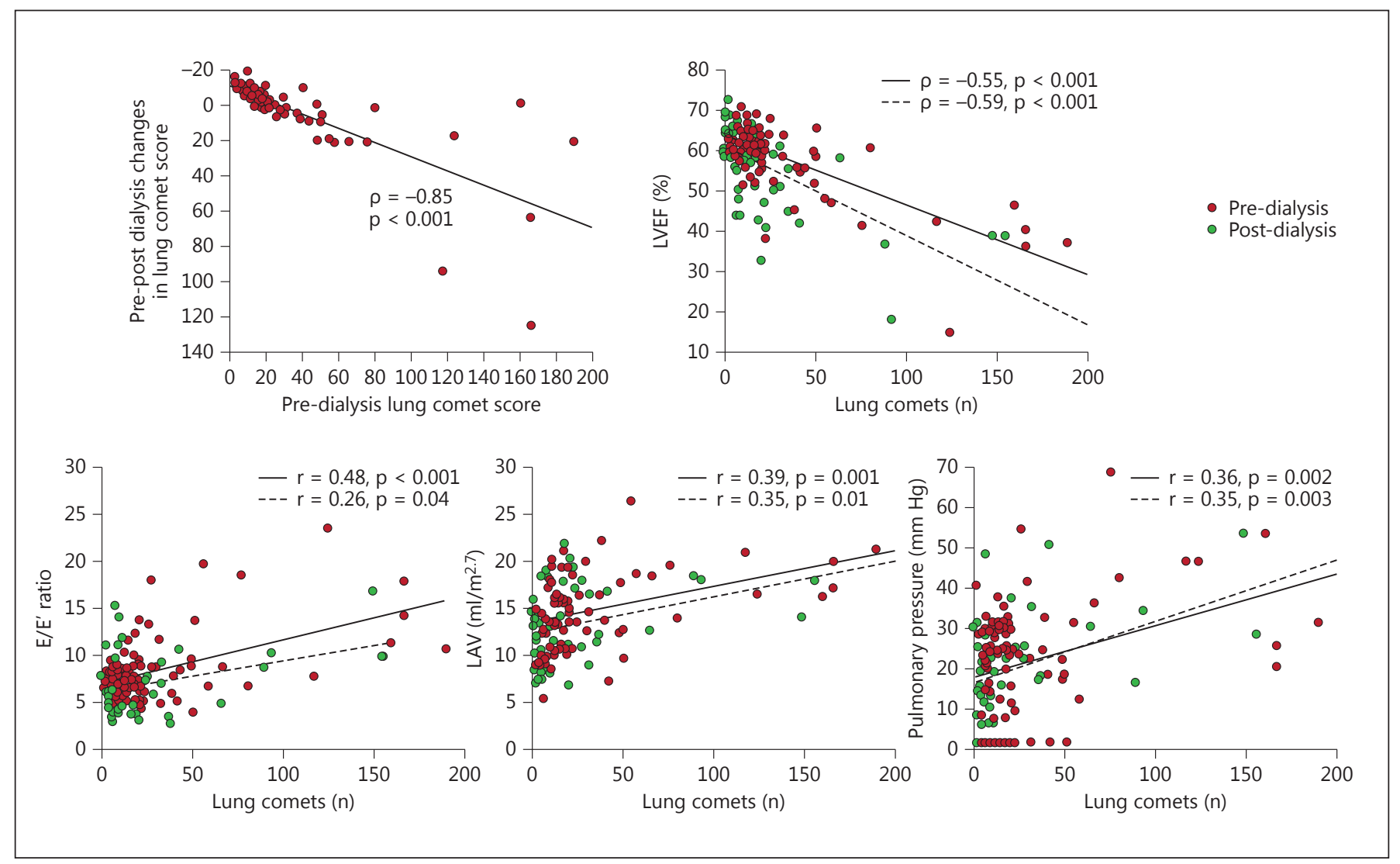

Fig. 3. Echocardiographic parameters of LV systolic (ejection fraction) and diastolic (E/E') function, left atrial volume and pulmonary pressure as related to US-B lines in HD patients before and after dialysis. The upper left panel depicts the relationship between the baseline number of US-B lines and the reduction in the number of the same parameter after dialysis (reproduced with permission by Elsevier [29]).

[34] and PD patients [35], lung congestion is not an innocent bystander which is strongly and independently associated with poor physical performance measured by the physical dimension item of the Short-Form 36 Quality Of Life Instrument. In the two studies where US lung water was measured along with total body water by bioimpedance analysis $[29,32]$, these two parameters were very weakly associated, which indicates that lung congestion can only partly be explained by volume overload. The scarce association between parameters of volume overload like total body water and US-B lines suggests that LV disorders play a major role in lung congestion in HD patients. This hypothesis is supported by the observation that US-B lines are closely related in an inverse fashion with parameters of systolic (ejection fraction) and diastolic $\left(E / E^{\prime}\right)$ function and in a direct fashion with left atrial volume and pulmonary pressure both before and after dialysis [29] (fig. 3). Pulmonary fibrosis may disturb the estimate of lung water by US because fibrosis generated US artifacts similar to US-B lines [26]. However, although the number of US-B lines is higher in HD patients with chronic obstructive pulmonary disease [32] than in HD patients without lung disease, in HD patients with chronic obstructive pulmonary disease a marked reduction in US-B lines also occurs across dialysis [29].

\section{Prognostic Relevance of Lung Congestion in ESRD}

The need of reliable biomarkers to monitor fluid volume in clinical practice is of utmost importance in research [4]. It is unquestionable that clinical trials are the final test to assess the clinical usefulness of any purported biomarker of volume expansion, an issue largely overlooked so far in clinical research in dialysis patients [4]. In the 'research ladder' aimed at establishing the clinical 


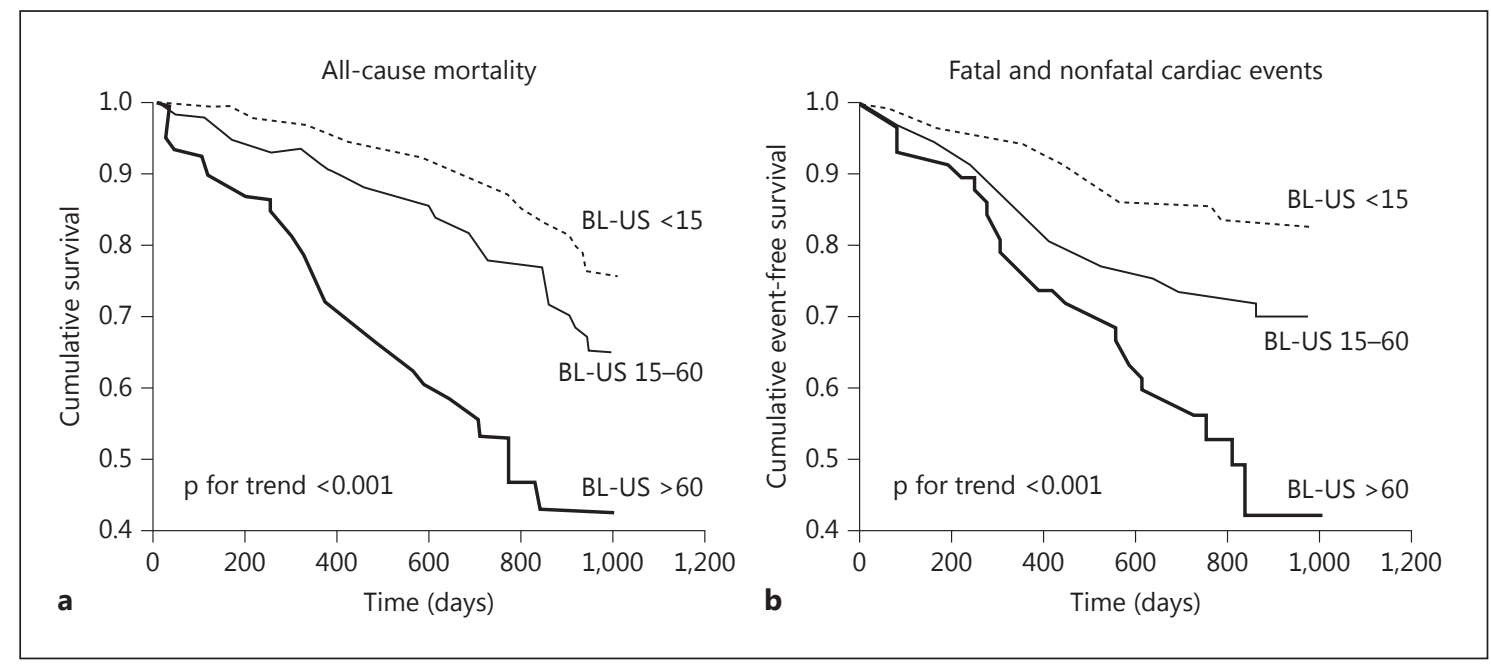

Fig. 4. Kaplan-Meyer survival (a) and cardiovascular event-free survival (b) in HD patients stratified according to the severity of lung congestion. $<15$ US-B lines (BL-US) = Mild or no congestion; $15-60$ BL-US = moderatesevere congestion; $>60$ BL-US = very severe congestion. Reproduced with permission of the American Society of Nephrology [31].

value of biomarkers, cohort studies represent an important, preliminary step to select worthy biomarkers deserving full-scale testing in a randomized clinical trial. The prognostic value of chest US for clinical outcome in patients with heart disease was tested in just two studies enrolling emergency patients admitted to a heart and lung department and in a sizable series of patients with coronary heart disease $[36,37]$. In both studies, chest US nicely predicted relevant clinical outcomes, including death and incident cardiovascular events. Of note, the predictive power of lung congestion in these studies was stronger than that of standard echocardiographic parameters, including ejection fraction, and independent of the NYHA score and classical risk factors such as diabetes [36]. When included in a three-variable echocardiographic score together with ejection fraction and tricuspid annular plane systolic excursion, US lung comets added prognostic value to an internationally established clinical score like the Global Registry in Acute Coronary Events score [37]. However important, these studies in patients with heart disease do not guarantee that chest US is prognostically useful in ESRD patients, a population with a unique risk profile [38]. Validation of biomarkers for prognosis needs to be performed in the precise population where the same biomarkers are purportedly useful for predicting relevant clinical events [39]. In this regard, in a recent study in 11 renal units in Italy, chest US added significant prognostic information for death and cardio- vascular events to classical risk factors, NYHA score, hypoalbuminemia, hyperphosphatemia and inflammation (fig. 4). Of note, this technique had important prognostic specificity because it significantly improved $(+10 \%)$ the reclassification of the risk for cardiac events in patients who remained free of cardiovascular events. Overall, these data suggest that the application of chest US may help to refine prognosis in dialysis patients [31]. Findings in this multicenter cohort study were replicated in a smaller single-center study in Romania [32]. Of relevance, in this study, chest US emerged as a stronger predictor of mortality than parameters of the hydration status measured by tetrapolar bioimpedance analysis, and this predictive power was largely independent of total body water and other bioimpedance parameters. Thus, important evidence is accumulating that chest US may become a useful adjunct to the diagnostic armamentarium of the modern clinical nephrologist.

\section{Clinical Perspectives}

Even though studies performed so far are quite consistent and methodologically valid, some limitations should be clearly acknowledged. Even though the multicenter study in Italy was fairly large and notwithstanding results in this study were confirmed in an external cohort in Romania [32], these studies included Caucasian patients 
only. Therefore, the prognostic ability of chest US should be confirmed in other dialysis populations including other ethnicities. Finally, the usefulness of chest US remains to be tested in a formal clinical trial. A clinical trial funded by the European Renal Association/European Dialysis and Transplant Association is underway to test the hypothesis that a lung comet-guided clinical policy may improve clinical outcome in high-risk HD patients with cardiac disease [40].

\section{References}

1 Blumberg A, Nelp WB, Hegstrom RM, Scribner BH: Extracellular volume in patients with chronic renal disease treated for hypertension by sodium restriction. Lancet 1967;ii:69-73.

$\checkmark 2$ Charra B, Chazot C: Volume control, blood pressure and cardiovascular function. Lessons from hemodialysis treatment. Nephron Physiol 2003;93:94-101.

3 Mees EJ: Volaemia and blood pressure in renal failure: have old truths been forgotten? Nephrol Dial Transplant 1995;10:1297-1298.

4 Agarwal R: Volume overload in dialysis: the elephant in the room, no one can see. Am J Nephrol 2013;38:75-77.

5 Arneson TJ, Liu J, Qiu Y, Gilbertson DT, Foley RN, Collins AJ: Hospital treatment for fluid overload in the Medicare hemodialysis population. Clin J Am Soc Nephrol 2010;5: 1054-1063.

6 6 Hecking M, Karaboyas A, Antlanger M, Saran R, Wizemann V, Chazot C, Rayner H, Horl WH, Pisoni RL, Robinson BM, Sunder-Plassmann G, Moissl U, Kotanko P, Levin NW, Saemann MD, Kalantar-Zadeh K, Port FK, Wabel P: Significance of interdialytic weight gain versus chronic volume overload: consensus opinion. Am J Nephrol 2013;38:78-90.

7 Kalantar-Zadeh K, Regidor DL, Kovesdy CP, Van WD, Bunnapradist S, Horwich TB, Fonarow GC: Fluid retention is associated with cardiovascular mortality in patients undergoing long-term hemodialysis. Circulation 2009;119:671-679.

-8 Parker TF III, Hakim R, Nissenson AR, Krishnan M, Bond TC, Chan K, Maddux FW, Glassock R: A quality initiative. Reducing rates of hospitalizations by objectively monitoring volume removal. Nephrol News Issues 2013;27:30-36.

9 Wizemann V, Wabel P, Chamney P, Zaluska W, Moissl U, Rode C, Malecka-Masalska T, Marcelli D: The mortality risk of overhydration in haemodialysis patients. Nephrol Dial Transplant 2009;24:1574-1579.

10 Agarwal R: Hypervolemia is associated with increased mortality among hemodialysis patients. Hypertension 2010;56:512-517.

-11 Tripepi G, Benedetto FA, Mallamaci F, Tripepi R, Malatino L, Zoccali C: Left atrial volume in end-stage renal disease: a prospective cohort study. J Hypertens 2006;24:11731180.
12 Reddan DN, Szczech LA, Hasselblad V, Lowrie EG, Lindsay RM, Himmelfarb J, Toto RD, Stivelman J, Winchester JF, Zillman LA, Califf RM, Owen WF Jr: Intradialytic blood volume monitoring in ambulatory hemodialysis patients: a randomized trial. J Am Soc Nephrol 2005; 16:2162-2169.

13 Zoccali C: How important is echocardiography for risk stratification in follow-up of patients with chronic kidney disease? Nat Clin Pract Nephrol 2007;3:178-179.

14 Shah MR, O'Connor CM, Sopko G, Hasselblad V, Califf RM, Stevenson LW: Evaluation Study of Congestive Heart Failure and Pulmonary Artery Catheterization Effectiveness (ESCAPE): design and rationale. Am Heart J 2001;141:528-535.

15 Crosbie WA, Snowden S, Parsons V: Changes in lung capillary permeability in renal failure. Br Med J 1972;iv:388-390.

16 Hoke TS, Douglas IS, Klein CL, He Z, Fang W, Thurman JM, Tao Y, Dursun B, Voelkel NF, Edelstein CL, Faubel S: Acute renal failure after bilateral nephrectomy is associated with cytokine-mediated pulmonary injury. J Am Soc Nephrol 2007;18:155-164.

17 Basnyat B, Murdoch DR: High-altitude illness. Lancet 2003;361:1967-1974.

18 Adamson PB, Magalski A, Braunschweig F, Bohm M, Reynolds D, Steinhaus D, Luby A, Linde C, Ryden L, Cremers B, Takle T, Bennett $\mathrm{T}$ : Ongoing right ventricular hemodynamics in heart failure: clinical value of measurements derived from an implantable monitoring system. J Am Coll Cardiol 2003;41: 565-571.

19 Yu CM, Wang L, Chau E, Chan RH, Kong SL, Tang MO, Christensen J, Stadler RW, Lau CP: Intrathoracic impedance monitoring in patients with heart failure: correlation with fluid status and feasibility of early warning preceding hospitalization. Circulation 2005;112: 841-848.

20 Trespalacios FC, Taylor AJ, Agodoa LY, Bakris GL, Abbott KC: Heart failure as a cause for hospitalization in chronic dialysis patients. Am J Kidney Dis 2003;41:1267-1277.

21 Parfrey PS, Harnett JD, Griffiths SM, Gault $\mathrm{MH}$, Barre PE: Congestive heart failure in dialysis patients. Arch Intern Med 1988;148: 1519-1525.
22 Zoccali C, Benedetto FA, Mallamaci F, Tripepi G, Giacone G, Cataliotti A, Seminara G, Stancanelli B, Malatino LS: Prognostic value of echocardiographic indicators of left ventricular systolic function in asymptomatic dialysis patients. J Am Soc Nephrol 2004;15:1029-1037.

-23 Foley RN, Parfrey PS, Harnett JD, Kent GM, Martin CJ, Murray DC, Barre PE: Clinical and echocardiographic disease in patients starting end-stage renal disease therapy. Kidney Int 1995;47:186-192.

24 Snashall PD, Weidner WJ, Staub NC: Extravascular lung water after extracellular fluid volume expansion in dogs. J Appl Physiol 1977;42:624-629.

25 Craddock PR, Fehr J, Brigham KL, Kronenberg RS, Jacob HS: Complement and leukocyte-mediated pulmonary dysfunction in hemodialysis. N Engl J Med 1977;296:769-774.

26 Volpicelli G, Elbarbary M, Blaivas M, Lichtenstein DA, Mathis G, Kirkpatrick AW, Melniker L, Gargani L, Noble VE, Via G, Dean A, Tsung JW, Soldati G, Copetti R, Bouhemad B, Reissig A, Agricola E, Rouby JJ, Arbelot C, Liteplo A, Sargsyan A, Silva F, Hoppmann R, Breitkreutz R, Seibel A, Neri L, Storti E, Petrovic $\mathrm{T}$ : International evidence-based recommendations for point-of-care lung ultrasound. Intensive Care Med 2012;38:577-591.

27 Fagenholz PJ, Gutman JA, Murray AF, Noble VE, Thomas SH, Harris NS: Chest ultrasonography for the diagnosis and monitoring of high-altitude pulmonary edema. Chest 2007; 131:1013-1018.

28 Picano E, Gargani L, Gheorghiade M: Why, when, and how to assess pulmonary congestion in heart failure: pathophysiological, clinical, and methodological implications. Heart Fail Rev 2010;15:63-72.

29 Mallamaci F, Benedetto FA, Tripepi R, Rastelli S, Castellino P, Tripepi G, Picano E, Zoccali C: Detection of pulmonary congestion by chest ultrasound in dialysis patients. JACC Cardiovasc Imaging 2010;3:586-594.

30 Noble VE, Murray AF, Capp R, Sylvia-Reardon $\mathrm{MH}$, Steele DJ, Liteplo A: Ultrasound assessment for extravascular lung water in patients undergoing hemodialysis. Time course for resolution. Chest 2009;135:1433-1439.

-31 Zoccali C, Torino C, Tripepi R, Tripepi G, D’Arrigo G, Postorino M, Gargani L, Sicari R, Picano E, Mallamaci F: Pulmonary congestion predicts cardiac events and mortality in ESRD. J Am Soc Nephrol 2013;24:639-646. 
32 Siriopol D, Hogas S, Voroneanu L, Onofriescu M, Apetrii M, Oleniuc M, Moscalu M, Sascau R, Covic A: Predicting mortality in haemodialysis patients: a comparison between lung ultrasonography, bioimpedance data and echocardiography parameters. Nephrol Dial Transplant 2013, E-pub ahead of print.

33 Panuccio V, Enia G, Tripepi R, Torino C, Garozzo M, Battaglia GG, Marcantoni C, Infantone L, Giordano G, De Giorgi ML, Lupia M, Bruzzese V, Zoccali C: Chest ultrasound and hidden lung congestion in peritoneal dialysis patients. Nephrol Dial Transplant 2012;27: 3601-3605.
34 Enia G, Torino C, Panuccio V, Tripepi R, Postorino M, Aliotta R, Bellantoni M, Tripepi G, Mallamaci F, Zoccali C: Asymptomatic pulmonary congestion and physical functioning in hemodialysis patients. Clin J Am Soc Nephrol 2013;8:1343-1348.

35 Enia G, Tripepi R, Panuccio V, Torino C, Garozzo M, Battaglia GG, Zoccali C: Pulmonary congestion and physical functioning in peritoneal dialysis patients. Perit Dial Int 2012;32: 531-536.

36 Frassi F, Gargani L, Tesorio P, Raciti M, Mottola G, Picano E: Prognostic value of extravascular lung water assessed with ultrasound lung comets by chest sonography in patients with dyspnea and/or chest pain. J Card Fail 2007;13:830-835.
Bedetti G, Gargani L, Sicari R, Gianfaldoni ML, Molinaro S, Picano E: Comparison of prognostic value of echographic [corrected] risk score with the Thrombolysis in Myocardial Infarction (TIMI) and Global Registry in Acute Coronary Events (GRACE) risk scores in acute coronary syndrome. Am J Cardiol 2010;106:1709-1716.

38 Zoccali C: Traditional and emerging cardiovascular and renal risk factors: an epidemiologic perspective. Kidney Int 2006;70:26-33.

39 Vasan RS: Biomarkers of cardiovascular disease: molecular basis and practical considerations. Circulation 2006;113:2335-2362.

40 Lust study (lung water by ultra-sound guided treatment to prevent death and cardiovascular complications in high risk ESRD patients with cardiomyopathy), in preparation. 